\title{
Operator connections and Borel measures on the unit interval
}

\author{
Pattrawut Chansangiam $^{\mathrm{a}}$, Wicharn Lewkeeratiyutkul ${ }^{\mathrm{b}, *}$ \\ a Department of Mathematics, Faculty of Science, King Mongkut's Institute of Technology Ladkrabang, \\ Bangkok 10520 Thailand \\ b Department of Mathematics and Computer Science, Faculty of Science, Chulalongkorn University, \\ Bangkok 10330 Thailand
}

*Corresponding author, e-mail: wicharn.1@chula.ac.th

Received 21 Jul 2014

Accepted 20 May 2015

\begin{abstract}
A connection is a binary operation for positive operators satisfying monotonicity, the transformer inequality, and joint-continuity from above. A normalized connection is called a mean. Here we show that there is a one-to-one correspondence between connections and finite Borel measures on the unit interval via the integral representation in terms of weighted harmonic means with respect to that measure. This correspondence is affine and order-preserving. Hence every mean can be regarded as an average of weighted harmonic means. We also investigate decompositions of connections, means, symmetric connections and symmetric means.
\end{abstract}

KEYWORDS: operator mean, operator monotone function, integral representation, weighted harmonic mean

MSC2010: 47A63 47A64 28B05

\section{INTRODUCTION}

An axiomatic theory of means for positive operators was given by Kubo and Ando $^{1}$ (see also Refs. 2-4). Consider the von Neumann algebra $B(\mathscr{H})$ of bounded linear operators on a Hilbert space $\mathscr{H}$. The set of positive operators on $\mathscr{H}$ is denoted by $B(\mathscr{H})^{+}$. For Hermitian operators $A, B \in$ $B(\mathscr{H})$, the partial order $A \leqslant B$ expresses the fact that $B-A \in B(\mathscr{H})^{+}$. A connection is a binary operation $\sigma$ on $B(\mathscr{H})^{+}$satisfying the following conditions for all positive operators $A, B, C, D$.

(M1) monotonicity:

$$
A \leqslant C, B \leqslant D \Longrightarrow A \sigma B \leqslant C \sigma D
$$

(M2) transformer inequality:

$$
C(A \sigma B) C \leqslant(C A C) \sigma(C B C)
$$

(M3) continuity from above: for $A_{n}, B_{n} \in B(\mathscr{H})^{+}$, if $A_{n} \downarrow A$ and $B_{n} \downarrow B$, then $A_{n} \sigma B_{n} \downarrow A \sigma B$. Here, $A_{n} \downarrow A$ indicates that $A_{n}$ is a decreasing sequence and $A_{n}$ converges strongly to $A$.

Two trivial examples are the left-trivial mean $(A, B) \mapsto A$ and the right-trivial mean $(A, B) \mapsto$ $B$. Typical examples of a connection are the sum $(A, B) \mapsto A+B$ and the parallel sum

$$
A: B=\left(A^{-1}+B^{-1}\right)^{-1}, \quad A, B>0 .
$$

The parallel sum was introduced by Anderson and Duffin $^{5}$ for analysing electrical networks. Recall that a multiport electrical network can be represented by a positive operator on a Hilbert space while the current through this network is described by the inner product on that space. The parallel sum $A: B$ is the total impedance of the network consisting of two subnetworks connected in parallel.

A mean is a connection $\sigma$ with normalized condition $I \sigma I=I$ or, equivalently, $A \sigma A=A$ for each $A \geqslant 0$. The class of Kubo-Ando means cover many well-known operator means in practice, e.g.,

(i) $t$-weighted arithmetic means:

$$
A \nabla_{t} B=(1-t) A+t B
$$

(ii) $t$-weighted geometric means:

$$
A \#_{t} B=A^{1 / 2}\left(A^{-1 / 2} B A^{-1 / 2}\right)^{t} A^{1 / 2}
$$

(iii) $t$-weighted harmonic means:

$$
A !_{t} B=\left[(1-t) A^{-1}+t B^{-1}\right]^{-1}
$$

(iv) logarithmic mean:

$$
(A, B) \mapsto A^{1 / 2} f\left(A^{-1 / 2} B A^{-1 / 2}\right) A^{1 / 2}
$$

where $f: \mathbb{R}^{+} \rightarrow \mathbb{R}^{+}, f(x)=(x-1) / \log x$. Here, $\mathbb{R}^{+}=[0, \infty)$. 
This axiomatic approach has applications in operator inequalities $s^{4,6,7}$, operator equations $s^{8,9}$ and operator entropy ${ }^{10}$.

A fundamental tool of the Kubo-Ando theory of connections and means is the theory of operator monotone functions. This concept was introduced in Ref. 11 (see also Refs. 12,13). A continuous real-valued function $f$ on an interval $I$ is called an operator monotone function if

$$
A \leqslant B \Longrightarrow f(A) \leqslant f(B)
$$

for all Hermitian operators $A, B \in B(\mathscr{H})$ whose spectra are contained in $I$ and for all Hilbert spaces $\mathscr{H}$.

A major core of the theory of connections and means is the interplay between connections, operator monotone functions, and Borel measures. Note first that if $\sigma$ and $\eta$ are connections and $k_{1}, k_{2} \in \mathbb{R}^{+}$, then the binary operation

$$
k_{1} \sigma+k_{2} \eta:(A, B) \mapsto k_{1}(A \sigma B)+k_{2}(A \eta B)
$$

is also a connection. The cone of connections on $B(\mathscr{H})^{+}$, the cone of operator monotone functions from $\mathbb{R}^{+}$to $\mathbb{R}^{+}$, and the cone of finite Borel measures on $[0, \infty]$ are equipped with pointwise partial orders. A connection $\sigma$ on $B(\mathscr{H})^{+}$can be characterized via operator monotone functions as follows.

Theorem 1 (Ref. 1) Given a connection $\sigma$, there is a unique operator monotone function $f: \mathbb{R}^{+} \rightarrow \mathbb{R}^{+}$ satisfying

$$
f(x) I=I \sigma(x I), \quad x \geqslant 0 .
$$

We call $f$ the representing function of $\sigma$. A connection has an integral representation with respect to a Borel measure.

Theorem 2 (Ref. 1) Given a connection $\sigma$, there is a unique finite Borel measure $\mu$ on $[0, \infty]$ such that

$$
A \sigma B=\alpha A+\beta B+\int_{(0, \infty)} \frac{\lambda+1}{\lambda}\{(\lambda A): B\} \mathrm{d} \mu(\lambda),
$$

for each $A, B \geqslant 0$. Here $\alpha=\mu(\{0\})$ and $\beta=\mu(\{\infty\})$.

In this paper, we characterize connections, means, symmetric connections and symmetric means in terms of Borel measures on the unit interval. Instead of the formula (1), we provide a meaningful integral representation as follows.
Theorem 3 Given a finite Borel measure $\mu$ on $[0,1]$, the binary operation

$$
A \sigma B=\int_{[0,1]} A !_{t} B \mathrm{~d} \mu(t), \quad A, B \geqslant 0
$$

is a connection on $B(\mathscr{H})^{+}$. The map $\mu \mapsto \sigma$ is bijective, affine and order-preserving, and the representing function of $\sigma$ is given by

$$
f(x)=\int_{[0,1]} 1 !_{t} x \mathrm{~d} \mu(t), \quad x \geqslant 0 .
$$

This theorem states that there is an isomorphism between connections and finite Borel measures on $[0,1]$ via a suitable integral representation. Moreover, a connection $\sigma$ is a mean if and only if $\mu$ is a probability measure. Hence every mean can be regarded as an average of weighted harmonic means. The weighted harmonic means form a building block for general connections. The dual of the logarithmic mean, defined by

$$
A \eta B=\left(B^{-1} \sigma A^{-1}\right)^{-1},
$$

where $\sigma$ denotes the logarithmic mean, provides an interesting example of our theory. In fact, this mean can be expressed as the integral of weighted harmonic means with respect to the Lebesgue measure on $[0,1]$.

Recall that a symmetric connection is a connection $\sigma$ such that $A \sigma B=B \sigma A$ for all $A, B \geqslant 0$. It follows that every symmetric connection admits an integral representation

$$
A \sigma B=\frac{1}{2} \int_{[0,1]}\left(A !_{t} B\right)+\left(B !_{t} A\right) \mathrm{d} \mu(t), A, B \geqslant 0
$$

for some finite Borel measure $\mu$ which is invariant under $\Theta:[0,1] \rightarrow[0,1], t \mapsto 1-t$.

We also investigate the structures of the set of finite Borel measures on $[0,1]$. This set is equipped with a suitable norm so that this cone becomes a normed cone. It is shown that this normed cone is isometrically isomorphic to the normed cone of connections.

The integral representation (2) also has advantages in treating decompositions of connections. It is shown that a connection $\sigma$ can be written as

$$
\sigma=\sigma_{\mathrm{ac}}+\sigma_{\mathrm{sd}}+\sigma_{\mathrm{sc}}
$$

where $\sigma_{\mathrm{ac}}, \sigma_{\mathrm{sd}}$ and $\sigma_{\mathrm{sc}}$ are connections. The 'singularly discrete part' $\sigma_{\text {sd }}$ is a countable sum of 
weighted harmonic means with nonnegative coefficients. The 'absolutely continuous part' $\sigma_{\mathrm{ac}}$ has an integral representation with respect to Lebesgue measure $m$ on $[0,1]$. The singularly continuous part' $\sigma_{\mathrm{sc}}$ has an integral representation with respect to a continuous measure mutually singular to $m$.

In the next section, we characterize connections in terms of finite Borel measures on $[0,1]$. The integral representations of practical operator connections with respect to finite Borel measures on $[0,1]$ are given in the third section. The fourth section deals with structures of the set of finite Borel measures and the set of connections. In the final section, we discuss decompositions of connections, means, symmetric connections and symmetric means.

\section{CONNECTIONS AND BOREL MEASURES ON THE UNIT INTERVAL}

In this section, we investigate the relationship between connections and Borel measures on $[0,1]$. Theorem 3 states that there is an affine correspondence between connections and finite Borel measures on $[0,1]$ via a suitable integral representation. To prove this fact, recall the following.

Lemma 1 (Ref. 11) A continuous function $f: \mathbb{R}^{+} \rightarrow$ $\mathbb{R}^{+}$is operator monotone if and only if there is a unique finite Borel measure $v$ on $[0, \infty]$ such that

$$
f(x)=\int_{[0, \infty]} \frac{x(\lambda+1)}{x+\lambda} \mathrm{d} v(\lambda), \quad x \geqslant 0 .
$$

Proof of Theorem 3: Since the family $\left\{A !_{t} B\right\}_{t \in[0,1]}$ is uniformly bounded by $\max \{\|A\|,\|B\|\}$ and $\mu([0,1])<\infty$, the binary operation (2) is welldefined. The monotonicity (M1) and the transformer inequality (M2) follow from passing these properties of weighted harmonic means through the integral. To show (M3), let $A_{n} \downarrow A$ and $B_{n} \downarrow B$. Then

$$
A_{n} !_{t} B_{n} \downarrow A !_{t} B .
$$

We obtain from the dominated convergence theorem that for each $\xi \in H$

$$
\begin{aligned}
\lim _{n \rightarrow \infty}\left\langle\left(A_{n} \sigma B_{n}\right) \xi, \xi\right\rangle & =\lim _{n \rightarrow \infty}\left\langle\int A_{n} !_{t} B_{n} \mathrm{~d} \mu(t) \xi, \xi\right\rangle \\
& =\int\left\langle\left(A !_{t} B\right) \xi, \xi\right\rangle \mathrm{d} \mu(t) \\
& =\left\langle\int A !_{t} B \mathrm{~d} \mu(t) \xi, \xi\right\rangle,
\end{aligned}
$$

i.e., $A_{n} \sigma B_{n} \downarrow A \sigma B$. Thus we have established a well-defined map $\mu \mapsto \sigma$. For the injectivity of this map, let $\mu_{1}$ and $\mu_{2}$ be finite Borel measures on $[0,1]$ such that

$$
\int_{0}^{1} A !_{t} B \mathrm{~d} \mu_{1}(t)=\int_{0}^{1} A !_{t} B \mathrm{~d} \mu_{2}(t), A, B \geqslant 0 .
$$

For each $i=1,2$ and $x \geqslant 0$, define

$$
f_{i}(x)=\int_{0}^{1} 1 !_{t} x \mathrm{~d} \mu_{i}(t)=\int_{0}^{\infty} \frac{x(\lambda+1)}{x+\lambda} \mathrm{d} \mu_{i} \Psi(\lambda),
$$

where $\Psi:[0, \infty] \rightarrow[0,1], t \mapsto t /(t+1)$ and the measure $\mu_{i} \Psi$ is defined by $E \mapsto \mu_{i}(\Psi(E))$ for each Borel set $E$. Then $f_{1}=f_{2}$ by setting $A=I$ and $B=x I$ for each $x \geqslant 0$. Lemma 1 implies that $\mu_{1}=\mu_{2}$.

For the surjectivity of this map, consider a connection $\sigma$. By Theorem 1 , there is a unique operator monotone function $f: \mathbb{R}^{+} \rightarrow \mathbb{R}^{+}$such that $f(x) I=I \sigma(x I)$ for $x \geqslant 0$. By Lemma 1 , there is a finite Borel measure $v$ on $[0, \infty]$ such that (5) holds. Define a finite Borel measure $\mu$ on $[0,1]$ by $\mu=\nu \Psi^{-1}$. Consider a connection

$$
\eta:(A, B) \mapsto \int_{[0,1]} A !_{t} B \mathrm{~d} \mu(t), \quad A, B \geqslant 0 .
$$

Then $I \eta(x I)=f(x) I$ for $x \geqslant 0$. Theorem 1 implies that $\eta=\sigma$. Hence the map $\mu \mapsto \sigma$ is surjective. This map is affine since $\mu$ is finite. It is easy to see that the map $\mu \mapsto \sigma$ is order preserving.

We call the measure $\mu$ the associated measure of a connection $\sigma$.

Remark 1 Even though the map $\mu \mapsto \sigma$ is order preserving, the inverse map $\sigma \mapsto \mu$ is not order preserving in general. For example, the associated measures of the harmonic mean $!_{1 / 2}$ and the arithmetic mean $\nabla_{1 / 2}=\left(!_{0}+!_{1}\right) / 2$ are given respectively by $\delta_{1 / 2}$ and $\left(\delta_{0}+\delta_{1}\right) / 2$. Here, $\delta_{t}$ denotes the Dirac measure at $t$. We have $!_{1 / 2} \leqslant \nabla_{1 / 2}$ but $\delta_{1 / 2} \nless$ $\left(\delta_{0}+\delta_{1}\right) / 2$.

Corollary 1 A connection is a mean if and only if its associated measure is a probability measure. Hence there is a one-to-one correspondence between means and probability Borel measures on $[0,1]$ via the integral representation (2).

Proof: The first assertion follows from the integral representation (2).

Hence every mean can be regarded as an average of special means, namely, weighted harmonic means. 
The weighted harmonic means form building blocks for general means.

The transpose of a connection $\sigma$ is the connection $(A, B) \mapsto B \sigma A$. Hence a connection is symmetric if and only if it coincides with its transpose. It was shown in Ref. 1 that if $f$ is the representing function of $\sigma$, then the representing function of the transpose of $\sigma$ is given by $x \mapsto x f(1 / x)$ for $x>0$.

Theorem 4 Let $\sigma$ be a connection with associated measure $\mu$. Then

(i) the representing function of the transpose of $\sigma$ is given by

$$
x \mapsto \int_{[0,1]} x !_{t} 1 \mathrm{~d} \mu(t), \quad x \geqslant 0 ;
$$

(ii) the associated measure of the transpose of $\sigma$ is given by $\mu \Theta^{-1}=\mu \Theta$ where

$$
\Theta:[0,1] \rightarrow[0,1], t \mapsto 1-t .
$$

Proof: The set function $\mu \Theta$ is clearly a finite Borel measure on $[0,1]$. Since the representing function $f$ of $\sigma$ is given by (3), the representing function of the transpose of $\sigma$ is given by

$$
x f\left(\frac{1}{x}\right)=x \int_{[0,1]} 1 !_{t} \frac{1}{x} \mathrm{~d} \mu(t)=\int_{[0,1]} x !_{t} 1 \mathrm{~d} \mu(t) .
$$

By changing the variable $t \mapsto 1-t$ and Theorem 3 , one can show that the associated measure of the transpose of $\sigma$ is $\mu \Theta$.

We say that a Borel measure $\mu$ on $[0,1]$ is symmetric if $\mu \Theta=\mu$. The next results follow from Theorems 3 and 4.

Corollary 2 There is a one-to-one correspondence between symmetric connections and finite symmetric Borel measures on $[0,1]$ via the integral representation

$$
A \sigma B=\frac{1}{2} \int_{[0,1]}\left(A !_{t} B\right)+\left(B !_{t} A\right) \mathrm{d} \mu(t), A, B \geqslant 0 .
$$

The representing function of the connection $\sigma$ in (7) can be written as

$$
f(x)=\frac{1}{2} \int_{[0,1]}\left(1 !_{t} x\right)+\left(x !_{t} 1\right) \mathrm{d} \mu(t), \quad x \geqslant 0
$$

and its associated measure is $\mu$. In particular, a connection is symmetric if and only if its associated measure is symmetric.
Corollary 3 There is a one-to-one correspondence between symmetric means and probability-symmetric Borel measures on the unit interval via the integral representation (7). A connection is a symmetric mean if and only if its associated measure is a probabilitysymmetric measure.

\section{CONCRETE EXAMPLES}

Example 1 The associated measure of the $t$ weighted harmonic mean $!_{t}$ is the Dirac measure $\delta_{t}$. In particular, the associated measures of the lefttrivial mean and the right-trivial mean are $\delta_{0}$ and $\delta_{1}$, respectively. By the affinity of the map $\mu \mapsto \sigma$, the associated measures of the sum and the parallel sum are given by $\delta_{0}+\delta_{1}$ and $\frac{1}{2} \delta_{1 / 2}$, respectively. Similarly, the $\alpha$-weighted arithmetic mean has the associated measure

$$
(1-\alpha) \delta_{0}+\alpha \delta_{1} .
$$

More generally, the measure $\sum_{i=1}^{n} a_{i} \delta_{t_{i}}$, where $t_{i} \in$ $[0,1]$ and $a_{i} \geqslant 0$, is associated with the connection $\sum_{i=1}^{n} a_{i} !_{t_{i}}$.

Recall that the Dirac measures are the extreme points of the convex set of probability Borel measures on $[0,1]$. Thus the weighted harmonic means are the extreme points of the convex set of means.

Example 2 Consider the $\alpha$-weighted geometric mean $\#_{\alpha}$ for $0<\alpha<1$. The representing function of this mean is given by $f(x)=x^{\alpha}$. A standard result from contour integration asserts that

$$
x^{\alpha}=\int_{[0, \infty]} \frac{x \lambda^{\alpha-1}}{x+\lambda} \frac{\sin \alpha \pi}{\pi} \mathrm{d} \lambda .
$$

It follows that the associated measure of $\#_{\alpha}$ is

$$
\mathrm{d} \mu(t)=\frac{\sin \alpha \pi}{\pi} \frac{1}{t^{1-\alpha}(1-t)^{\alpha}} \mathrm{d} t .
$$

Example 3 Let us compute the associated measure of the logarithmic mean. Recall that the representing function of this mean is the operator monotone function $f(x)=(x-1) / \log x$. Then by Example 2 we have

$$
\begin{aligned}
f(x) & =\int_{0}^{1} x^{\lambda} \mathrm{d} \lambda \\
& =\int_{0}^{1} \int_{0}^{1} \frac{\sin \lambda \pi}{\pi} \frac{1 !_{t} x}{t^{1-\lambda}(1-t)^{\lambda}} \mathrm{d} t \mathrm{~d} \lambda \\
& =\int_{0}^{1}\left(1 !_{t} x\right) \int_{0}^{1} \frac{\sin \lambda \pi}{\pi t^{1-\lambda}(1-t)^{\lambda}} \mathrm{d} \lambda \mathrm{d} t .
\end{aligned}
$$


Hence the associated measure of the logarithmic mean has density $g$ given by

$$
g(t)=\frac{1}{t(1-t)\left(\pi^{2}+\log ^{2}\left(\frac{t}{1-t}\right)\right)} .
$$

Example 4 Consider the dual of the logarithmic mean defined by (4). Its representing function is given by $x \mapsto(x \log x) /(x-1)$. It turns out that its associated measure is the Lebesgue measure on $[0,1]$, i.e.,

$$
A \eta B=\int_{[0,1]} A !_{t} B \mathrm{~d} t .
$$

\section{THE ISOMETRIC ISOMORPHISM BETWEEN CONNECTIONS AND BOREL MEASURES}

In this section, we establish an isometric isomorphism between the normed cone of connections and the normed cone of finite Borel measures on $[0,1]$.

Denote by $C\left(B(\mathscr{H})^{+}\right)$the set of connections on $B(\mathscr{H})^{+}$. This set is a cone with respect to usual addition and scalar multiplication in which the neutral element is the zero connection $(A, B) \mapsto 0$. In Ref. 14 , this cone is equipped with a norm given by

$$
\|\sigma\|=\sup \{\|A \sigma B\|: A, B \geqslant 0,\|A\|=\|B\|=1\}
$$

for each connection $\sigma$. In fact, $\|\sigma\|=\|I \sigma I\|$. The pair $\left(C\left(B(\mathscr{H})^{+}\right),\|\cdot\|\right)$ becomes a normed cone.

The set of finite Borel measures on $[0,1]$ is written as $B M([0,1])$. This set is a cone with respect to usual addition and scalar multiplication in which the neutral element is the zero measure. A natural norm for each measure in this cone is defined by its total variation:

$$
\|\mu\|=\mu([0,1]) .
$$

The cone $B M([0,1])$ is also a normed cone.

Given any normed cone $(C,\|\cdot\|)$, we can equip it with a 1st-countable topology ${ }^{14}$ defined by a pseudo-metric

$$
d: C \times C \rightarrow \mathbb{R}^{+}, \quad d(x, y)=|\|x\|-\|y\|| .
$$

A function $\varphi: C \rightarrow D$ between normed cones is called an isomorphism if it is a continuous affine bijection whose inverse is continuous. By an isometry, we mean a function $\phi: C \rightarrow D$ such that

$$
\|\phi(x)\|=\|x\| \quad \text { for all } x \in C .
$$

Note that every isometry between normed cones is continuous and injective. The inverse of an isometry is also an isometry. If $\varphi: C \rightarrow D$ is an isomorphism which is also an isometry, we say that $\varphi$ is an isometric isomorphism. In this case, $C$ is said to be isometrically isomorphic to $D$.

Theorem 5 The normed cones $C\left(B(\mathscr{H})^{+}\right)$and $B M([0,1])$ are isometrically isomorphic via the isometric isomorphism $\sigma \mapsto \mu_{\sigma}$, where $\mu_{\sigma}$ is the representing measure of $\sigma$.

Proof: The function $\Psi: \sigma \mapsto \mu_{\sigma}$ is an affine bijection by Theorem 3. For each connection $\sigma$, we have

$$
I \sigma I=\int_{[0,1]} I !_{t} I \mathrm{~d} \mu(t)=\mu([0,1]) I
$$

and hence

$$
\|\Psi(\sigma)\|=\left\|\mu_{\sigma}\right\|=\mu([0,1])=\|I \sigma I\|=\|\sigma\| .
$$

Thus $\Psi$ is an isometric isomorphism.

Consequently, a connection is a mean if and only if its norm or the norm of its associated measure is 1 . The cone of symmetric connections is isometrically isomorphic to the cone of symmetric finite Borel measures on $[0,1]$.

\section{DECOMPOSITIONS OF CONNECTIONS}

This section deals with decompositions of connections, means, symmetric connections, and symmetric means.

Theorem 6 Let $\sigma$ be a connection on $B(\mathscr{H})^{+}$. Then there is a unique triple $\left(\sigma_{\mathrm{ac}}, \sigma_{\mathrm{sc}}, \sigma_{\mathrm{sd}}\right)$ of connections on $B(\mathscr{H})^{+}$such that

$$
\sigma=\sigma_{\mathrm{ac}}+\sigma_{\mathrm{sc}}+\sigma_{\mathrm{sd}}
$$

and

(i) $\sigma_{\text {sd }}$ is a countable sum of weighted harmonic means with nonnegative coefficients, i.e., there are a countable set $D \subseteq[0,1]$ and a family $\left\{a_{t}\right\}_{t \in D} \subseteq \mathbb{R}^{+}$such that $\sum_{t \in D} a_{t}<\infty$ and

$$
\sigma_{\mathrm{sd}}=\sum_{t \in D} a_{t} !_{t},
$$

i.e., for each $A, B \geqslant 0$,

$$
A \sigma_{\mathrm{sd}} B=\sum_{t \in D} a_{t}\left(A !{ }_{t} B\right)
$$

and the series converges in the norm topology;

(ii) there is a (unique m-a.e.) integrable function $g$ : $[0,1] \rightarrow \mathbb{R}^{+}$such that

$$
A \sigma_{\mathrm{ac}} B=\int_{[0,1]} g(t)\left(A !_{t} B\right) \mathrm{d} m(t), \quad A, B \geqslant 0
$$


(iii) its associated measure of $\sigma_{\mathrm{sc}}$ is continuous and mutually singular to $m$.

Moreover, the representing functions of $\sigma_{\mathrm{ac}}, \sigma_{\mathrm{sd}}$ and $\sigma_{\mathrm{sc}}$ are given respectively by

$$
\begin{aligned}
& f_{\mathrm{ac}}(x)=\int_{[0,1]} 1 !_{t} x \mathrm{~d} \mu_{\mathrm{ac}}, \\
& f_{\mathrm{sd}}(x)=\int_{[0,1]} 1 !_{t} x \mathrm{~d} \mu_{\mathrm{sd}}=\sum_{t \in D} a_{t}\left(1 !_{t} x\right) \\
& f_{\mathrm{sc}}(x)=\int_{[0,1]} 1 !_{t} x \mathrm{~d} \mu_{\mathrm{sc}}
\end{aligned}
$$

and the associated measure of $\sigma_{\mathrm{sd}}$ is given by $\sum_{t \in D} a_{t} \delta_{t}$.

Proof: Let $\mu$ be the associated measure of $\sigma$. By a refinement of Lebesgue decomposition for a Borel measure on $\mathbb{R}$ with respect to Lebesgue measure $m$ (see, e.g., Ref. 15), there is a unique triple $\left(\mu_{\mathrm{ac}}, \mu_{\mathrm{sc}}, \mu_{\mathrm{sd}}\right)$ of finite Borel measures on $[0,1]$ such that

$$
\mu=\mu_{\mathrm{ac}}+\mu_{\mathrm{sc}}+\mu_{\mathrm{sd}}
$$

where $\mu_{\mathrm{sd}}$ is a discrete measure, $\mu_{\mathrm{ac}}$ is absolutely continuous with respect to $m$, and $\mu_{\mathrm{sc}}$ is a continuous measure mutually singular to $m$. Define

$$
\begin{aligned}
A \sigma_{\mathrm{ac}} B & =\int_{[0,1]} A !_{t} B \mathrm{~d} \mu_{\mathrm{ac}}(t), \\
A \sigma_{\mathrm{sc}} B & =\int_{[0,1]} A !_{t} B \mathrm{~d} \mu_{\mathrm{sc}}(t), \\
A \sigma_{\mathrm{sd}} B & =\int_{[0,1]} A !_{t} B \mathrm{~d} \mu_{\mathrm{sd}}(t)=\sum_{t \in D} a_{t}\left(A !_{t} B\right)
\end{aligned}
$$

for each $A, B \geqslant 0$. The series $\sum_{t \in D} a_{t}\left(A !_{t} B\right)$ converges in the norm topology. Indeed, the fact that, for each $n<m$ in $\mathbb{N}$ and $t_{i} \in[0,1]$,

$$
\begin{gathered}
\left\|\sum_{i=1}^{n} a_{t_{i}}\left(A !_{t_{i}} B\right)-\sum_{i=1}^{m} a_{t_{i}}\left(A !_{t_{i}} B\right)\right\| \\
\leqslant \sum_{i=n+1}^{m} a_{t_{i}}\left\|A !_{t_{i}} B\right\| \\
\leqslant \sum_{i=n+1}^{m} a_{t_{i}}\left(\|A\| !_{t_{i}}\|B\|\right) \\
\leqslant \sum_{i=n+1}^{m} a_{t_{i}} \max \{\|A\|,\|B\|\}
\end{gathered}
$$

together with the condition $\sum_{i=1}^{\infty} a_{t_{i}}<\infty$ implies the convergence of the series $\sum_{i=1}^{\infty} a_{t_{i}}\left(A !_{t_{i}} B\right)$.
The one-to-one correspondence between operator monotone functions on $\mathbb{R}^{+}$and finite Borel measures on $[0,1]$ shows that the associated measures of $\sigma_{\mathrm{ac}}, \sigma_{\mathrm{sd}}$, and $\sigma_{\mathrm{sc}}$ are given by $\mu_{\mathrm{ac}}, \mu_{\mathrm{sd}}$, and $\mu_{\mathrm{sc}}$, respectively. The rest of theorem comes from Theorem 3 and the fact that the associated measure of $!_{t}$ is $\delta_{t}$ for each $t \in[0,1]$ in Example 1 .

This theorem states that every connection $\sigma$ consists of three parts. The 'singularly discrete part' $\sigma_{\text {sd }}$ is a countable sum of weighted harmonic means. This type of connection includes the weighted arithmetic means, the sum, and the parallel sum. The 'absolutely continuous part' $\sigma_{\mathrm{ac}}$ arises as an integral with respect to the Lebesgue measure, given by (9). Examples 2, 3, and 4 show that the weighted geometric means, the logarithmic mean, and its dual are typical examples of such connections. The 'singularly continuous part' $\sigma_{\mathrm{sc}}$ has an integral representation with respect to a continuous measure mutually singular to the Lebesgue measure. Hence (aside from the singularly continuous part) this theorem gives an explicit description of general connections.

Proposition 1 Consider the connection $\sigma_{\mathrm{ac}}$ defined by (9). Then

(i) it is symmetric if and only if $g \circ \Theta=g$;

(ii) it is a mean if and only if the average of the density function $g$ is 1 , i.e., $\int_{0}^{1} g(t) \mathrm{d} t=1$.

Proof: Use Corollaries 1 and 2.

We say that a density function $g:[0,1] \rightarrow \mathbb{R}^{+}$is symmetric if $g \circ \Theta=g$. Next, we decompose a mean as a convex combination of means.

Corollary 4 Let $\sigma$ be a mean on $B(\mathscr{H})^{+}$. Then there are unique triples $\left(\tilde{\sigma}_{\mathrm{ac}}, \tilde{\sigma}_{\mathrm{sc}}, \tilde{\sigma}_{\mathrm{sd}}\right)$ of means or zero connections on $B(\mathscr{H})^{+}$and $\left(k_{\mathrm{ac}}, k_{\mathrm{sc}}, k_{\mathrm{sd}}\right)$ of real numbers in $[0,1]$ such that

$$
\sigma=k_{\mathrm{ac}} \tilde{\sigma}_{\mathrm{ac}}+k_{\mathrm{sc}} \tilde{\sigma}_{\mathrm{sc}}+k_{\mathrm{sd}} \tilde{\sigma}_{\mathrm{sd}}, \quad k_{\mathrm{ac}}+k_{\mathrm{sc}}+k_{\mathrm{sd}}=1
$$

and

(i) there are a countable set $D \subseteq[0,1]$ and a family $\left\{a_{t}\right\}_{t \in D} \subseteq \mathbb{R}^{+}$such that $\sum_{t \in D} a_{t}=1$ and $\tilde{\sigma}_{s d}=$ $\sum_{t \in D} a_{t} !_{t}$;

(ii) there is a (unique m-a.e.) integrable function $g$ : $[0,1] \rightarrow \mathbb{R}^{+}$with average 1 such that

$$
A \tilde{\sigma}_{\mathrm{ac}} B=\int_{[0,1]} g(t)\left(A !_{t} B\right) \mathrm{d} m(t), \quad A, B \geqslant 0 ;
$$

(iii) its associated measure of $\tilde{\sigma}_{\mathrm{sc}}$ is continuous and mutually singular to $\mathrm{m}$. 
Proof: Let $\mu$ be the associated probability measure of $\sigma=\sigma_{\mathrm{ac}}+\sigma_{\mathrm{sd}}+\sigma_{\mathrm{sc}}$ and we write $\mu=\mu_{\mathrm{ac}}+\mu_{\mathrm{sd}}+\mu_{\mathrm{sc}}$. Suppose that $\mu_{\mathrm{ac}}, \mu_{\mathrm{sd}}$ and $\mu_{\mathrm{sc}}$ are non-zero. Then

$$
\begin{aligned}
\mu=\mu_{\mathrm{ac}}([0,1]) \frac{\mu_{\mathrm{ac}}}{\mu_{\mathrm{ac}}([0,1])}+ & \mu_{\mathrm{sd}}([0,1]) \frac{\mu_{\mathrm{sd}}}{\mu_{\mathrm{sd}}([0,1])} \\
& +\mu_{\mathrm{sc}}([0,1]) \frac{\mu_{\mathrm{sc}}}{\mu_{\mathrm{sc}}([0,1])} .
\end{aligned}
$$

Set

$$
\begin{aligned}
& k_{\mathrm{ac}}=\mu_{\mathrm{ac}}([0,1]), k_{\mathrm{sd}}=\mu_{\mathrm{sd}}([0,1]), k_{\mathrm{sc}}=\mu_{\mathrm{sc}}([0,1]), \\
& \tilde{\mu}_{\mathrm{ac}}=\frac{\mu_{\mathrm{ac}}}{k_{\mathrm{ac}}}, \tilde{\mu}_{\mathrm{sd}}=\frac{\mu_{\mathrm{sd}}}{k_{\mathrm{sd}}}, \tilde{\mu}_{\mathrm{sc}}=\frac{\mu_{\mathrm{sc}}}{k_{\mathrm{sc}}} .
\end{aligned}
$$

Define $\tilde{\sigma}_{\mathrm{ac}}, \tilde{\sigma}_{\mathrm{sd}}, \tilde{\sigma}_{\mathrm{sc}}$ to be the means corresponding to the measures $\tilde{\mu}_{\mathrm{ac}}, \tilde{\mu}_{\mathrm{sd}}, \tilde{\mu}_{\mathrm{sc}}$, respectively. Now apply Theorem 6 and Proposition 1.

We can decompose a symmetric connection as a nonnegative linear combination of symmetric connections as follows.

Corollary 5 Let $\sigma$ be a symmetric connection on $B(\mathscr{H})^{+}$. Then there is a unique triple $\left(\sigma_{\mathrm{ac}}, \sigma_{\mathrm{sc}}, \sigma_{\mathrm{sd}}\right)$ of symmetric connections on $B(\mathscr{H})^{+}$such that

$$
\sigma=\sigma_{\mathrm{ac}}+\sigma_{\mathrm{sc}}+\sigma_{\mathrm{sd}}
$$

and

(i) there is a countable set $D \subseteq[0,1]$ and a family $\left\{a_{t}\right\}_{t \in D} \subseteq \mathbb{R}^{+}$such that $a_{t}=a_{1-t}$ for all $t \in D$, $\sum_{t \in D} a_{t}<\infty$ and $\sigma_{\mathrm{sd}}=\sum_{t \in D} a_{t} !_{t}$

(ii) there is a (unique m-a.e.) symmetric integrable function $g:[0,1] \rightarrow \mathbb{R}^{+}$such that

$$
\begin{gathered}
A \sigma_{\mathrm{ac}} B=\frac{1}{2} \int_{[0,1]} g(t)\left(A !_{t} B+B !_{t} A\right) \mathrm{d} m(t), \\
A, B \geqslant 0 ;
\end{gathered}
$$

(iii) its associated measure of $\sigma_{\mathrm{sc}}$ is continuous and mutually singular to $m$.

Proof: Let $\mu$ be the associated measure of $\sigma$ and decompose $\mu=\mu_{\mathrm{ac}}+\mu_{\mathrm{sd}}+\mu_{\mathrm{sc}}$ where $\mu_{\mathrm{ac}} \ll m$, $\mu_{\mathrm{sd}}$ is a discrete measure, $\mu_{\mathrm{sc}}$ is continuous, and $\mu_{\mathrm{sc}} \perp \mathrm{m}$. Then $\mu \Theta=\mu_{\mathrm{ac}} \Theta+\mu_{\mathrm{sd}} \Theta+\mu_{\mathrm{sc}} \Theta$. It is straightforward to show that $\mu_{\mathrm{ac}} \Theta<m, \mu_{\mathrm{sd}} \Theta$ is discrete, $\mu_{\mathrm{sc}} \Theta$ is continuous and $\mu_{\mathrm{sc}} \Theta \perp \mathrm{m}$. Corollary 2 implies that $\mu \Theta=\mu$. The uniqueness of the decomposition of measures implies that

$$
\mu_{\mathrm{ac}} \Theta=\mu_{\mathrm{ac}}, \mu_{\mathrm{sd}} \Theta=\mu_{\mathrm{sd}} \text { and } \mu_{\mathrm{sc}} \Theta=\mu_{\mathrm{sc}} .
$$

Again, Corollary 2 tells us that $\sigma_{\mathrm{ac}}, \sigma_{\mathrm{sd}}$, and $\sigma_{\mathrm{sc}}$ are symmetric connections. The rest of the proof follows from Theorem 6 and Proposition 1.
A decomposition of a symmetric mean as a convex combination of symmetric means is obtained by normalizing symmetric connections as in the proof of Corollary 4.

\section{REFERENCES}

1. Kubo F, Ando T (1980) Means of positive linear operators. Math Ann 246, 205-24.

2. Chansangiam P, Lewkeeratiyutkul W (2013) Characterizations of connections for positive operators. Southeast Asian Bull Math 37, 1-13.

3. Hiai F (2010) Matrix analysis: matrix monotone functions, matrix means, and majorizations. Interdiscipl Inform Sci 16, 139-248.

4. Chansangiam P (2012) Operator means and applications. In: Yasser HA (ed) Linear Algebra-Theorems and Applications, InTech, Rijeka, pp 163-88.

5. Anderson WN, Duffin RJ (1969) Series and parallel addition of matrices. J Math Anal Appl 26, 576-94.

6. Ando T (1979) Concavity of certain maps on positive definite matrices and applications to Hadamard products. Lin Algebra Appl 26, 203-41.

7. Mond B, Pečarić J, Šunde J, Varošanec S (1997) Operator versions of some classical inequalities. Lin Algebra Appl 246, 117-26.

8. Anderson WN, Morley T, Trapp G (1990) Positive solutions to $X=A-B X^{-1} B$. Lin Algebra Appl 134, 53-62.

9. Lim Y (2008) On Ando-Li-Mathias geometric mean equations. Lin Algebra Appl 428, 1767-77.

10. Fujii J (1992) Operator means and the relative operator entropy. Oper Theory Adv Appl 59, 161-72.

11. Löwner C (1934) Über monotone matrix Funktionen. Math Z 38, 177-216.

12. Bhatia R (1997) Matrix Analysis, Springer-Verlag, New York.

13. Hiai F, Yanagi K (1995) Hilbert spaces and linear operators, Makino Pub. Ltd

14. Chansangiam P, Lewkeeratiyutkul W (2013) The normed ordered cone of operator connections. Chamchuri J Math 5, 45-55.

15. Folland GB (1999) Real Analysis: Modern Techniques and Their Applications, 2nd edn, Wiley, New York. 